\title{
Longer observation time increases adenoma detection in the proximal colon - a prospective study
}

\section{다)(요 $\odot$}

\author{
Authors \\ Peter Klare ${ }^{1}$, Henrik Phlipsen ${ }^{1}$, Bernhard Haller ${ }^{2}$, Henrik Einwächter ${ }^{1}$, Andreas Weber ${ }^{1}$, Mohamed Abdelhafez ${ }^{1}$, \\ Monther Bajbouj', Hayley Brown', Roland M. Schmid'1, Stefan von Delius'
}

Institutions

1 II. Medizinische Klinik, Klinikum rechts der Isar der Technischen Universität München, Munich, Germany

2 Institut für Medizinische Statistik und Epidemiologie, Klinikum rechts der Isar der Technischen Universität München, Munich, Germany

submitted 3.4.2017

accepted after revision 8.9.2017

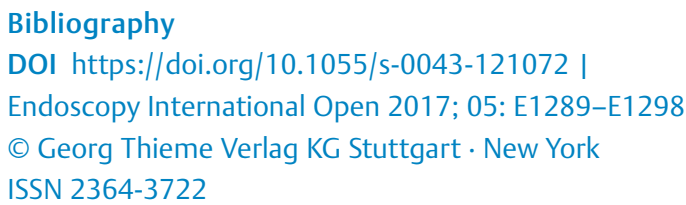

Corresponding author

Peter Klare, MD, II. Medizinische Klinik, Klinikum rechts der Isar, Ismaninger Str. 22, 81675 München, Germany Fax: +498941404905 peter.klare@tum.de

\section{ABSTRACT}

Background and study aims Longer observation times are associated with increased adenoma detection rates $(A D R)$ in the entire colon. However, adenomas in the proximal colon are at risk of being missed during colonoscopy. The aim of this study was to investigate the impact of observation time on detection of adenomatous polyps in the proximal colon.
Patients and methods This was a prospective study at a university hospital in Germany. Colonoscopies were conducted using magnetic endoscope imaging (MEI) in order to determine the exact position of the scope. Exact observation times spent for the detection of polyps in the proximal and distal colon segments were assessed. The primary outcome was adenoma detection in the proximal colon. ROC curves were generated in order to test the correlation between observation time and adenoma detection. Logistic regression analysis was used to check for interfering factors.

Results A total 480 procedures with 538 polyps were available for analysis. The overall adenoma detection rate was $38.5 \%$. ADR in the proximal colon was $28.0 \%$. There was a significant association between observation time in the proximal colon and the detection of proximal adenomas $(P$ $<0.001)$. The impact of the time factor on ADR was stronger in the proximal compared to the distal colon $(P=0.030)$. A net period of $4 \mathrm{~min} 7 \mathrm{sec}$ was found to be the minimum time span for sufficient adenoma detection in the proximal colon.

Conclusion Observation time is significant in terms of adenoma detection in the proximal colon. The impact of observation time on ADR is stronger in the proximal compared to the distal colon. In the proximal colon a minimum time span of 4 minutes should be spent in order to ensure adequate adenoma detection.

ClinicalTrials.gov identifier: NCT02819492

\section{Introduction}

Endoscopic adenoma resection is the most effective strategy to prevent colorectal cancer (CRC). Since the establishment of screening programs declining CRC incidence and mortality rates have been observed in the United States [1], the UK [2] and Europe [3]. However the effect of screening seems to be limited with respect to the incidence of proximal colorectal cancer $[4,5]$. Insufficient detection of proximal adenomas could be one possible explanation for higher proximal CRC inci- dences. Indeed studies showed that proximal adenomas are at risk of being missed during screening colonoscopy $[6,7]$. In general spending enough time for polyp screening during colonoscopy is a crucial factor in order to sufficiently detect adenomas. A withdrawal time of at least 6 minutes has become an important quality criterion for screening investigations. However the respective threshold is only valid for the entire colon. No specifications are available regarding observation time spans with respect to the proximal and distal colon sections. 
A careful investigation is mandatory for the detection of any lesion regardless of its location. However the texture of lesions existing at the respective colon segments might differ leading to different difficulty levels of detection. Until now it is unclear whether the duration of withdrawal is essential for adenoma detection equally throughout the colon or whether side-specific differences exist.

In this prospective study we therefore investigated the impact of observation time on adenoma detection in the proximal colon.

\section{Patients and methods}

\section{Patients}

The study protocol was approved by the local ethics committees and registered at ClinicalTrials.gov (Identification Number: NCT02819492). Outpatients and inpatients aged $\geq 40$ years scheduled for colonoscopy at the study site (II. Medizinische Klinik und Poliklinik, Klinikum rechts der Isar der Technischen Universität München, Munich) were eligible to participate. Exclusion criteria were emergency examinations, American Society of Anesthesiologists risk classes IV and above, pregnant women, inflammatory bowel disease (IBD), polyposis syndromes, recently detected but unresected polyps and contraindications for polyp removal. Written informed consent was obtained from all patients.

\section{Study design and colonoscopy procedure}

This was an open label prospective study at a university hospital in Germany. Patients received polyethylene glycol (PEG) for bowel cleansing. The standard regimen consisted of two liters PEG administered as split dose prior to colonoscopy. A total of 20 experienced endoscopists (minimum experience of 200 independently conducted investigations) participated in the trial. All investigations were conducted using Olympus Evis Exera ${ }^{\circledR}$ III CF-HQ 190 colonoscopes (Olympus, Tokio, Japan). The highdefinition white light modus of the endoscopes was used for polyp screening throughout the investigation. In case of polyp detection NBI mode was additionally used for polyp characterization. Cecal intubation time was defined as the time span from insertion of the scope into the anus until reaching the appendix orifice. The withdrawal time was defined as the time span spent for withdrawing the scope from the cecum until extracting the scope from the anus. In order to assess the exact observation time during withdrawal, we measured time exposure spent for polyp resection, biopsy sampling and other concrete actions not associated with polyp detection by using a stopwatch. The required time was then subtracted from the withdrawal time in order to determine the exact observation time during withdrawal. Time exposure spent for flushing and air insufflation was not subtracted from withdrawal time. In order to determine the localization of the scope in the colon a magnetic endoscope imaging (MEI) device (ScopeGuide ${ }^{\circledR}$, Olympus, Tokyo, Japan) was switched on throughout the colonoscopy procedures. The device provides a real-time $3 \mathrm{D}$ image of the shape and position of the endoscope [8]. The $3 \mathrm{D}$ image was integrated in the lower left part of the monitor where it was displayed throughout the procedure. Immediately after insertion of the colonoscope into the anus the MEI device was calibrated. Images provided by the MEI device have been shown to correlate well with anatomic conditions being displayed by x-ray imaging [9]. The MEl system was also used to assess the exact localization of detected polyps and to assign them to either the proximal or the distal colon. The proximal (right) colon was defined as the area of the colon from the cecum up to and including the splenic flexure. All polyps found in this part were grouped under the term "proximal (right-sided) polyps". Polyps found further down were assigned to the distal (left) colon. We recorded number, localization and size of detected polyps and tumors. Tumors were defined as exophytic ulcerated lesions in this trial. The size of the polyps was estimated by comparing the polyp with the open biopsy forceps. According to current guidelines, polyps up to $5 \mathrm{~mm}$ in size were resected using the biopsy forceps [10]. Resection was performed using a resection snare if polyps were larger than $5 \mathrm{~mm}$.

Propofol (Braun, Melsungen, Germany) or a combination of propofol and midazolam (Braun, Melsungen, Germany) was used for sedation. All patients were monitored for the duration of the procedure. Their blood pressure was measured every 5 minutes, a pulse oximetry was used and an electrocardiogram was used in selected cases in patients with preexisting cardiac disease. All patients received $4 \mathrm{~L} /$ min oxygen via a nasal cannula throughout the procedure. After colonoscopy patients were disconnected from electronic monitoring and transferred to the recovery area provided they had gained an adequate level of consciousness.

\section{Pathological polyp evaluation}

All polyps were resected and sent to pathology for histopathological examination. All pathologists were board certified. Due to internal quality assurance, polyps were consecutively investigated by two internal pathologists. Polyps revealing serrated adenoma histology were considered adenomas in this trial.

\section{Endpoints}

Primary endpoint was the detection of adenomatous polyps in the proximal part of the colon. Secondary endpoints were (i) polyp and adenoma detection rates in the distal colon, (ii) polyp morphology and (iii) procedural characteristics including observation time in the respective colon sections.

\section{Sample size calculation}

For the association of observation time with adenoma detection an odds ratio for the standardized observation time was assumed to be 1.5 translating to a regression coefficient in the logistic regression of 0.405 (based on own unpublished data). It was assumed that adenomas could be detected in $13 \%$ of all patients in the proximal colon at the mean observation time [11]. Sample size calculation was performed with nQuery Advisor version 7.0 . 
- Table 1 Demographic, clinical, and procedural characteristics.

\begin{tabular}{|l|c|}
\hline Factor & \\
\hline Age (y), mean (SD) & $63.5(10.5)$ \\
\hline Male sex & $248(51.7 \%)$ \\
\hline Body mass index (kg/m²), mean (SD) & $26.1(7.3)$ \\
\hline Smoking (current and ex) & $477 / 478(99.8 \%)$ \\
\hline Alcohol abuse & $99(20.6 \%)$ \\
\hline Diabetes mellitus & $88(18.3 \%)$ \\
\hline ASA class & $61(12.7 \%)$ \\
\hline - 1 & \\
\hline - 2 & $145(30.3 \%)$ \\
\hline - 3 & $237(49.5 \%)$ \\
\hline Ambulatory patients & $97(20.3 \%)$ \\
\hline Indications & $282(58.8 \%)$ \\
\hline - Screening & \\
\hline - Grounds to suspect tumor & $170(35.4 \%)$ \\
\hline - Gastrointestinal bleeding or anemia & $92(19.2 \%)$ \\
\hline - Abdominal complaints & $73(15.2 \%)$ \\
\hline - History of colorectal polyps & $104(21.7 \%)$ \\
\hline - Surveillance after colorectal carcinoma & $14(2.9 \%)$ \\
\hline - Others & $14(2.9 \%)$ \\
\hline in that category. Data were fully available unless otherwise noted. \\
\hline
\end{tabular}

\section{Statistical analysis}

Continuous data are described by mean and standard deviation (mean $\pm \mathrm{sd}$ ) or median, first and third quartile (median [1st quartile - 3 rd quartile]), respectively, for categorical data absolute and relative frequencies are presented. For assessment of the association between observation time and adenoma detection, a receiver operating characteristics (ROC) analysis was performed. The area under the ROC curve (AUC) and a corresponding $95 \%$ confidence interval are presented. A proposal for a minimum observation time was derived based on the Youden index. Analyses were performed separately for proximal and distal colon section and AUCs between the colon sections were compared using Delong's test. Additionally, a univariate and a multivariable logistic regression model was fit to the data. Adenoma detection (yes $=1$, no $=0$ ) was used as dependent variable and the observation time (after logarithmic transformation due to its skewed distribution) was considered as independent variable. In the multivariable regression model, additionally the potential confounders gender, age, diabetes, BMI, alcohol abuse, nicotine abuse, indication for colonoscopy and

\begin{tabular}{|c|c|c|}
\hline \multicolumn{3}{|c|}{ Assessed for eligibility $(n=619)$} \\
\hline$\rightarrow$ & $\begin{array}{l}\text { Excluded } \\
\text { Not meeting eligibility criteria } \\
\text { Refused to participate } \\
\text { Others reasons }\end{array}$ & $\begin{array}{r}(n=119) \\
(n=84) \\
(n=4) \\
(n=31)\end{array}$ \\
\hline \multicolumn{3}{|c|}{ Enrollment $(n=500)$} \\
\hline$\rightarrow$ & $\begin{array}{l}\text { Did not receive complete investigation } \\
\text { Poor bowel preparation } \\
\text { Stenosis } \\
\text { Others reasons }\end{array}$ & $\begin{array}{r}(n=20) \\
(n=11) \\
(n=5) \\
(n=4)\end{array}$ \\
\hline \multicolumn{3}{|c|}{ Analysis } \\
\hline \multicolumn{3}{|l|}{$\downarrow$} \\
\hline $\operatorname{lnv}$ & stigations suitable for analysis $(n=480)$ & \\
\hline
\end{tabular}

Fig. 1 Patient flow through the trial.

cecal intubation time were included in the model. In order to adjust for different colonoscopy indications, indications described in - Table 1 were entered as categorical variables in the logistic regression model for assessment of association between time and adenoma detection rate. For comparison of resected polyps between distal and proximal colon generalized estimation equation (GEE) models were fit to the data as multiple polyps were resected in some patients. Logistic GEE models were used for binary outcomes (e.g. type of polyp), linear GEE models for quantitative outcomes (polyp size). Due to the skewed distribution of polyp size a logarithmic transformation was used. An exchangeable correlation structure was considered for all models. A $P$ value $<0.05$ was considered statistically significant. Statistical analysis was performed using statistical software R (R Foundation for Statistical Computing, Vienna, Austria) version 3.3.1 [12].

\section{Results}

A total of 619 patients were screened for eligibility. In 84 cases patients did not meet the inclusion criteria and 4 did not give written consent. In a further 31 patients, inclusion was not possible for other reasons (e.g. procedures took place in examination rooms where the MEI function unit was not installed) ( $\triangleright$ Fig. 1). A total of 500 patients were included in the study. Of the 500 participants, 20 were later excluded from the analysis as colonoscopy procedures were terminated prematurely due to poor bowel preparation (11 cases), stenosis (five cases) or other reasons for failed incomplete colonoscopy (four cases). In no case was premature termination of the procedure due to the patients' participation in the study. Thus the final analysis contained 480 patients. 
- Table 2 Procedural data obtained from 480 colonoscopies.

\begin{tabular}{|l|c|}
\hline Factor & \\
\hline $\begin{array}{l}\text { Total duration of colonoscopy } \\
\text { median (1st quartile }-3 \text { rd quartile) }\end{array}$ & $10: 00(21: 30-44: 00)$ \\
\hline $\begin{array}{l}\text { Cecal intubation time in minutes } \\
\text { median (1st quartile }-3 \text { rd quartile) }\end{array}$ & $10: 00(6: 00-16: 00)$ \\
\hline $\begin{array}{l}\text { Total observation time } \\
\text { median (1st quartile }-3 \text { rd quartile) }\end{array}$ & $8: 28(6: 45-11: 38)$ \\
\hline $\begin{array}{l}\text { Observation time in the proximal colon } \\
\text { median (1st quartile }-3 \text { rd quartile) }\end{array}$ & $4: 00(3: 00-5: 31)$ \\
\hline $\begin{array}{l}\text { Observation time in the distal colon } \\
\text { median (0.25 - } 0.75 \text { quantile) }\end{array}$ & $4: 00(3: 00-6: 00)$ \\
\hline $\begin{array}{l}\text { Boston Bowel Preparation Score } \\
\text { median (min - max) }\end{array}$ & $8(2-9)$ \\
\hline $\begin{array}{l}\text { Total propofol dose in mg } \\
\text { median (min-max) }\end{array}$ & $1873 / 480(98.5 \%)$ \\
\hline $\begin{array}{l}\text { Values are presented as median (1st quartile to } 3 \text { rd quartile) or median } \\
\text { (minimum - maximum). } \\
\text { Small numbers indicate proportion of patients for whom data was available } \\
\text { in that category. Data were fully available unless otherwise noted. }\end{array}$ \\
\hline
\end{tabular}

\section{Patients and procedural characteristics}

Patient characteristics of 480 analysed cases are shown in $>$ Table 1. Characteristics of the 20 patients excluded from the final analysis are given in the supplement.

The median duration of procedures was 31 minutes (Inter quartile range, IQR: 1 st quartile: $21: 30 \mathrm{~min}$ to 3 rd quartile: 44:00 min) ( $\triangleright$ Table 2 ). The median cecal intubation time was 10 minutes (IQR 6:00 min to 16:00 min) minutes. After subtraction of time exposure spent for actions not connected with polyp detection (e.g. polypectomy or biopsy sampling), the median pure observation time was 8:28 minutes (IQR 6:45 min to $11: 38 \mathrm{~min}$ ) in the entire colon. The median pure observation time in the proximal colon was 4:00 minutes (IQR 3:00 min to 5:31 min). The respective observation time was 4:00 min (IQR 3:00 min to $6: 00 \mathrm{~min}$ ) in the distal colon. Procedural data obtained from the 480 procedures are given in $>$ Table 2 .

\section{Detection of polyps and tumors}

In the 480 patients analyzed a total of 570 polyps were detected by 20 different colonoscopists. Eighteen polyps were not resected because of relative contraindications for polypectomy (e.g. simultaneous detection of tumors). Eight polyps were lost during the process of resection and extraction from the coIon. Histopathological diagnoses of a further 6 polyps were not available due to other reasons. Thus a total of 538 resected polyps with available pathological diagnoses were analysed in this study. The median polyp size was $4 \mathrm{~mm}$ (IQR: $2 \mathrm{~mm}$ to 6 $\mathrm{mm})$. Total polyp detection rate (PDR) and adenoma detection rate (ADR) were $54.0 \%$ and $38.5 \%$, respectively. Twelve large ulcerated tumors were detected in 11 patients. Biopsy samples taken from tumors showed carcinomatous histology in all 11 cases. Among 538 polyps histopathological diagnoses showed
330 (61.3\%) adenomatous and 206 (38.3\%) non-adenomatous polyps (e.g. hyperplastic polyps, inflammatory polyps). Two resected polyps $(0.4 \%)$ showed carcinomatous histology. Both cases received a multidisciplinary tumor board review immediately after histopathological diagnoses were reported. Morphological and histopathological polyp features are shown in the Supplement.

Of the 538 polyps 288 were in the proximal colon and 250 were in the distal colon. Adenoma detection rates in the respective colon segments were $27.7 \%$ and $18.3 \%$. Adenomatous histology was diagnosed more frequently in proximal polyps (75.3\%) compared to distal polyps (45.2\%) $(P<0.001)$. Morphology of polyps differed between the two colon segments. More pedunculated polyps were found in the distal colon (15 $(6.2 \%)$ vs. $6(2.1 \%), P=0.047)$. Polyp size did not differ significantly between the two colon segments (median polyp size = $4 \mathrm{~mm}$, IQR $2 \mathrm{~mm}$ to $6 \mathrm{~mm}$ for both segments, $P=0.789$ ). Nine colonoscopists contributed 409 of the 480 (85.2\%) investigations to this trial. Personal ADRs of these 9 key colonoscopists ranged between $24 \%$ and $50 \%$ in this study.

\section{Impact of observation time on the detection of proximal adenomas}

- Fig. 2 shows the estimated probability of adenoma detection in the proximal colon. Time specific proximal ADR values measured in this trial are given in $>$ Table 3.

There was a significant association between longer observation time and the detection of adenomas in the proximal colon $(P<0.001)$. The area under the ROC curve was $0.68(95 \% \mathrm{Cl}$ $0.62-0.73$ ) ( Fig.3). Fig. 4 shows the observation time spans of the proximal colon in patients being diagnosed with and without adenomas (at least one adenomatous polyp in the proximal colon segment). Calculation of the Youden index revealed a minimum proximal observation time span of 4:07 minutes in this cohort. In $68.4 \%$ of patients with detected proximal adenomas, colonoscopists spent at least 4:07 minutes observing the proximal colon section while in $66.3 \%$ of the patients without detected adenomas the observation time was less than 4:07 minutes.

\section{Impact of observation time on the detection of distal adenomas}

The estimated probability of adenoma detection in the distal colon is shown in $\mathbf{F i g}$. 5 . The respective time-specific ADR values are given in $>$ Table 3 .

There was a significant association between longer observation time and the detection of adenomas in the distal colon $(P=$ 0.020). The area under the ROC curve was 0.58 (95\% Cl $0.51-$ 0.65) ( $\triangleright$ Fig.3). $>$ Fig. 6 shows observation time spans of the distal colon in patients being diagnosed with and without adenomas.

Based on calculation of the Youden index the minimum observation time span was found to be 4:02 minutes in the distal colon. In $63.6 \%$ of patients being diagnosed with distal adenomas, colonoscopists spent at least 4:02 minutes observing the distal colon section while in $55.1 \%$ of the patients without de- 


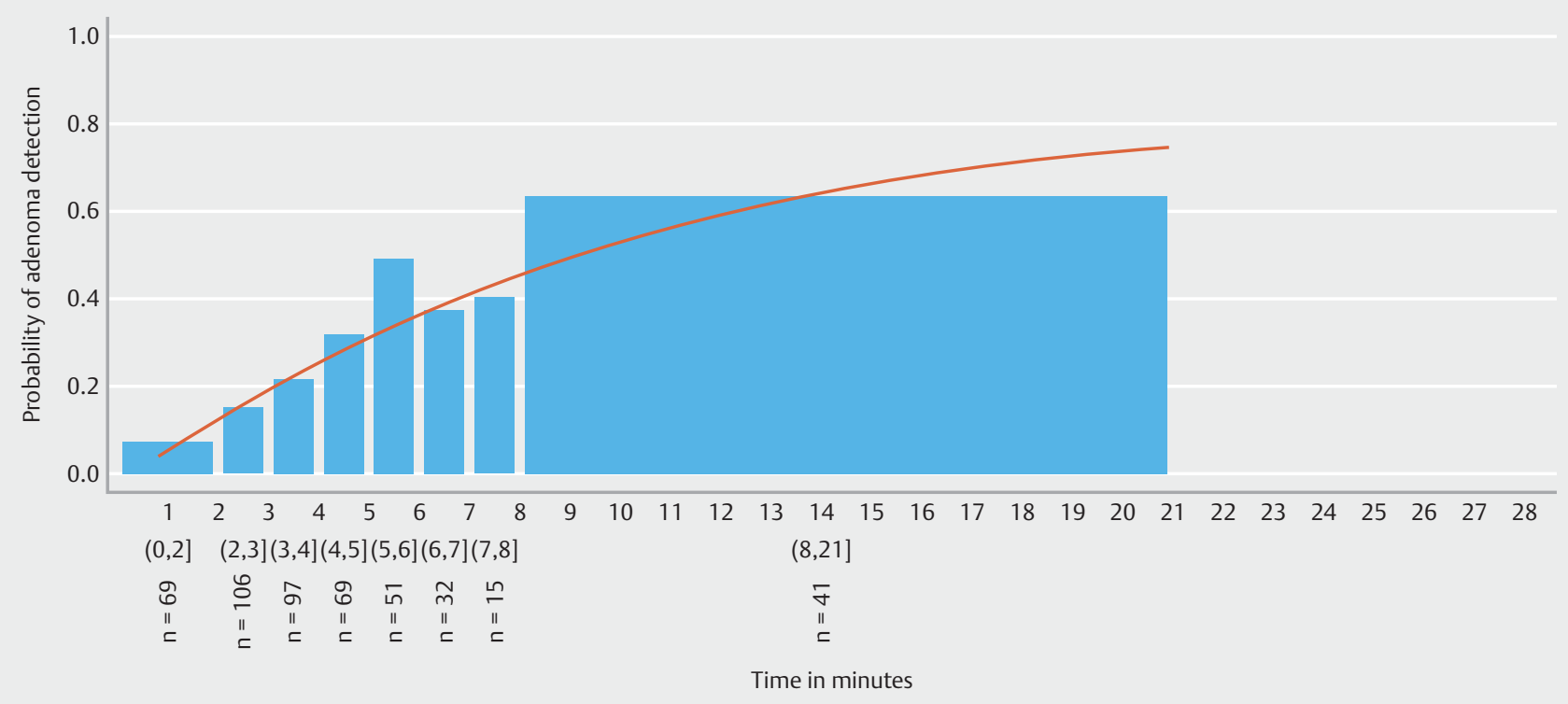

Fig. 2 Estimated probability of adenoma detection in the proximal colon. Round brackets signify included and square brackets signify excluded time values of the respective time spans.

- Table 3 Side-specific adenoma detection rates according to gradually rising observation time spans in the respective colon segments.

\begin{tabular}{|l|l|l|}
\hline $\begin{array}{l}\text { Observation time in the respective } \\
\text { segment (proximal/distal) (min) }\end{array}$ & $\begin{array}{l}\text { Proximal } \\
\text { ADR }\end{array}$ & $\begin{array}{l}\text { Distal } \\
\text { ADR }\end{array}$ \\
\hline$(0-2]$ & $7.2 \%$ & $8.8 \%$ \\
\hline$(2-3]$ & $15.1 \%$ & $13.4 \%$ \\
\hline$(3-4]$ & $21.6 \%$ & $15.3 \%$ \\
\hline$(4-5]$ & $31.9 \%$ & $22.1 \%$ \\
\hline$(5-6]$ & $49.0 \%$ & $17.4 \%$ \\
\hline$(6-7]$ & $37.5 \%$ & $18.6 \%$ \\
\hline$(7-8]$ & $40.0 \%$ & $29.6 \%$ \\
\hline$>8$ & $63.4 \%$ & $43.3 \%$ \\
\hline
\end{tabular}

Round brackets signify included and square brackets signify excluded time values of the respective time spans.

$\mathrm{ADR}$, adenoma detection rate.

tected adenomas the observation time was less than 4:02 minutes.

\section{Impact of time effect on ARD - comparison between proximal and distal colon}

The association of observation time and adenoma detection was stronger in the proximal colon compared to the distal colon $(P=0.030)$. A comparison of the area under ROC curves is given in $>$ Fig. 3.

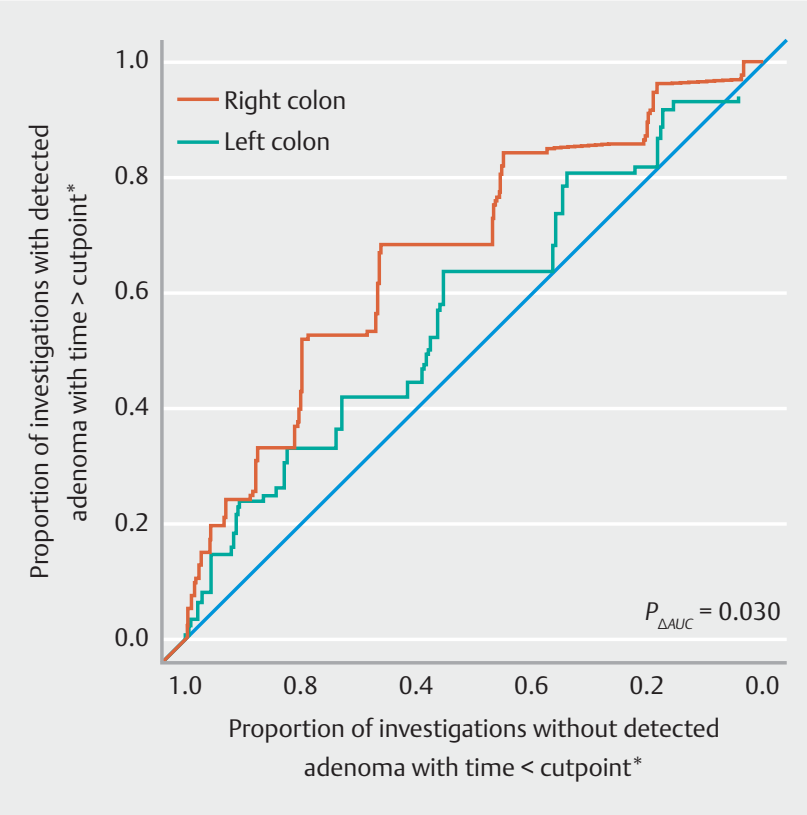

Fig. 3 Receiver-operating characteristic (ROC) curve for the performance of adenoma detection according to observation time in the proximal and distal colon. For the proximal colon the area under the ROC curve was 0.68 ( $95 \% \mathrm{Cl} 0.62-0.73)$. For the distal colon the area under ROC curve was $0.58(95 \% \mathrm{Cl} 0.51-0.65)$.

${ }^{*}$ As in common ROC analysis all possible cutpoints for investigation time derived from the data were considered and combinations of resulting proportions are illustrated in the figure.

\section{Impact of the time effect in screening patients}

After exclusion of patients receiving colonoscopy for other reasons than screening 170 patients were available for analysis. In this sub-analysis a significant association between longer ob- 


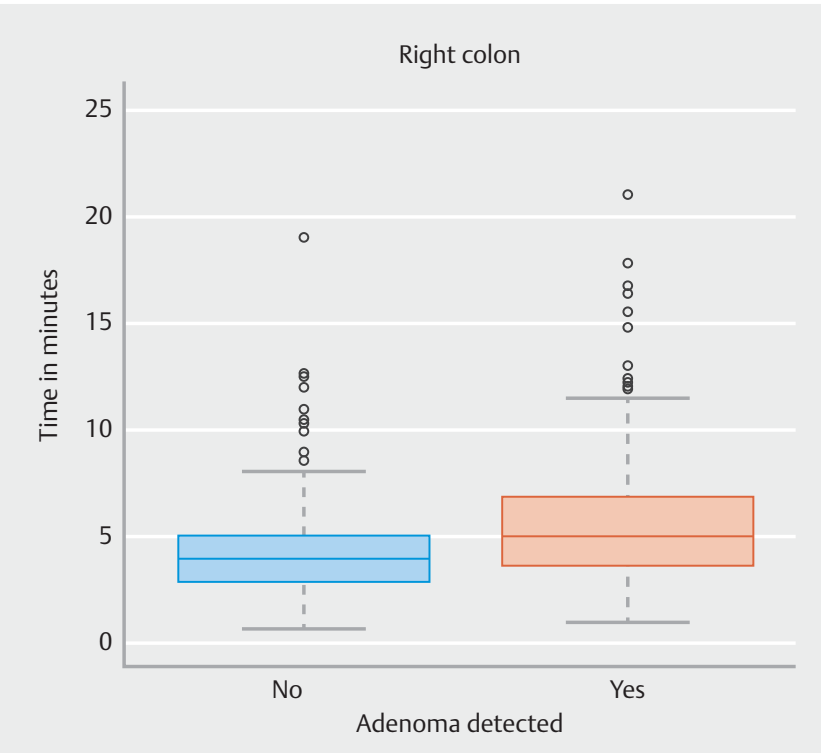

- Fig. 4 Proximal observation time in patients being diagnosed with and without proximal adenomas.

servation time and the detection of adenomas in the proximal colon was found $(P<0.001)$. The area under the ROC curve was $0.74(95 \% \mathrm{Cl} 0.66-0.82)$. Calculation for the distal colon showed no significant association between longer observation time and the detection of adenomas in the screening subgroup $(p=0.398)$. The area under the ROC curve for the distal colon was 0.55 (95\% Cl $0.43-0.67)$.

\section{Logistic regression analysis}

\section{Proximal colon}

A longer observation time was associated with the detection of adenomas in the proximal colon. A difference of one unit in the logarithmized observation time was associated with an odds ratio of 3.643 (95\% Cl $2.370-5.599 ; P<0.001)$. Univariate analysis showed male gender $(P=0.023)$, advanced age $(P<0.001)$ and alcohol abuse $(P=0.046)$ to significantly predict for adenoma detection. After controlling for potential confounders (gender, age, diabetes, BMI, alcohol or nicotine abuse, indication for colonoscopy, (log-)cecal intubation time) (log-)observation time was still significantly associated with adenoma detection in the proximal colon (OR 3.610, 95\% Cl 2.383-5.709, $P<$ 0.001) ( Table 4).

\section{Distal colon}

Univariate regression analysis showed that longer observation time was a predictor for adenoma detection in the distal colon. (Log-)observation time was significantly associated with the chance to detect adenomatous polyps (OR $=1.811(95 \% \mathrm{Cl}$ $1.145-2.888, P=0.011)$. In addition advanced patient age predicted adenoma detection in the univariate setting $(P=0.007)$. Indication for colonoscopy "bleeding/anemia" was a factor that prevented detection of adenomas in the univariate analysis $(P=$ 0.015). After controlling for confounders, (log-)observation time remained an independent predictor for adenoma detection in the distal colon (OR 2.341, 95\% Cl 1.395-3.929, $P=$ 0.001) ( $\triangleright$ Table 4).

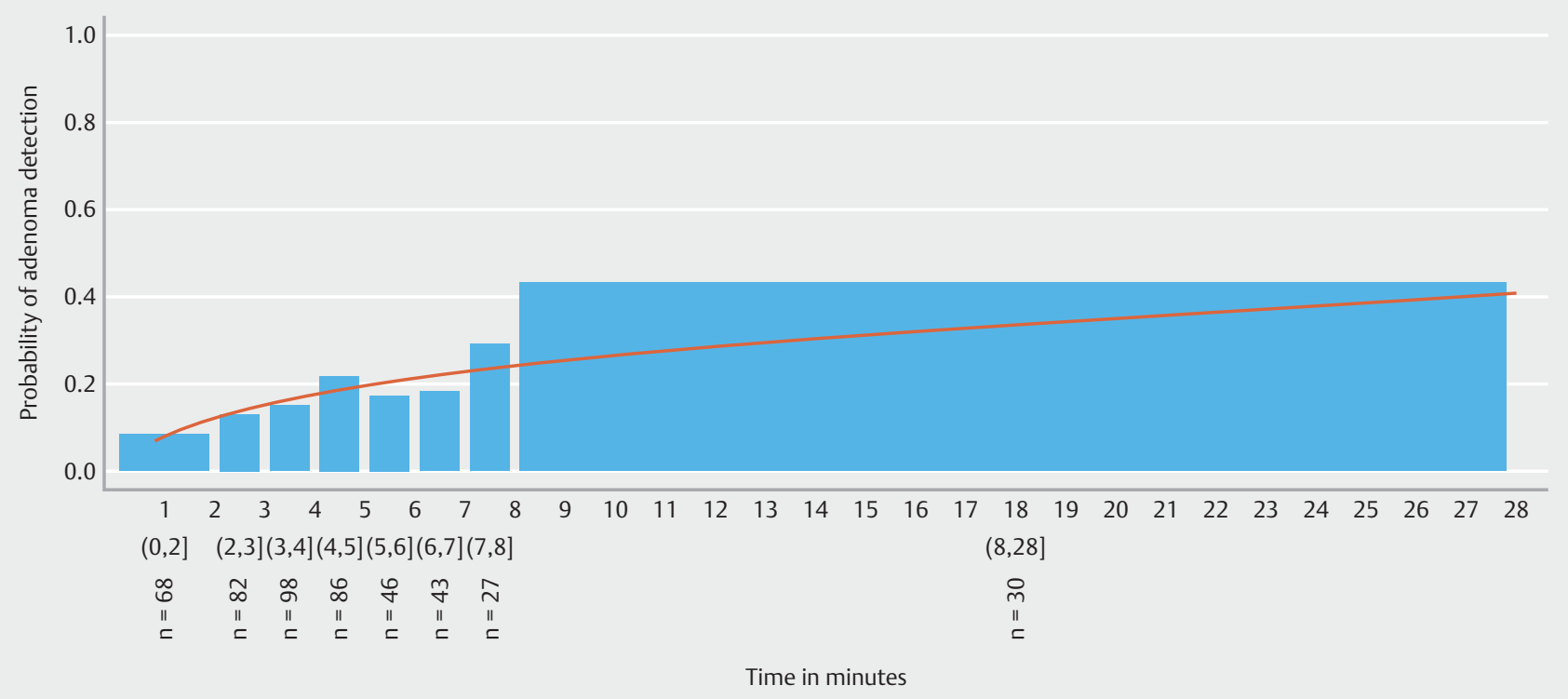

- Fig. 5 Estimated probability of adenoma detection in the distal colon. Round brackets signify included and square brackets signify excluded time values of the respective time spans. 


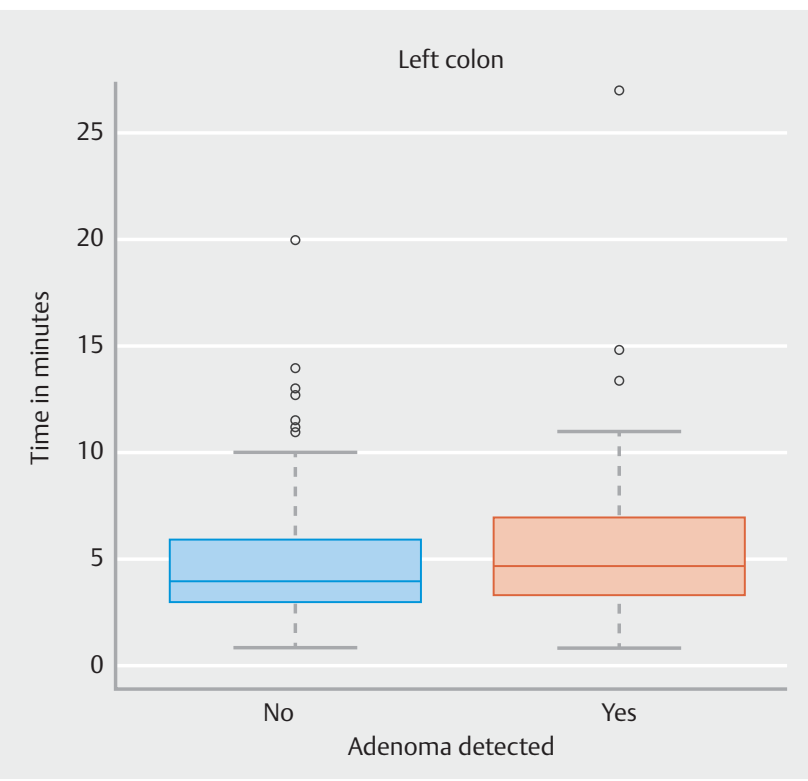

- Fig. 6 Distal observation time in patients being diagnosed with and without distal adenomas.

\section{Serious adverse events}

There were no serious adverse events observed during the trial.

\section{Discussion}

The detection of adenomatous lesions is the key task of screening colonoscopy. A multitude of procedural factors have been shown to influence the ADR in clinical trials [13]. In order to detect adenomas sufficiently, colonoscopists need to spend an adequate amount of time searching the colon mucosa. To date a withdrawal time of at least 6 minutes has become a quality parameter for screening colonoscopy $[14,15]$. However it has been shown that proximal polyps are at risk of being missed during colonoscopy $[6,7]$. The latter fact may contribute to a limited success of screening colonoscopy to prevent proximal CRC. A proximal tumor location has been determined as a predictor for interval carcinomas [16]. Moreover tumor registry data suggest that improvements of proximal CRC outcome are low compared to improvements regarding distal CRC mortality
[17]. Thus reasonable measures should be taken in order to increase the detection of proximal precursor lesions during colonoscopy.

\section{Time aspect is more important in the proximal than in the distal colon}

In this study we showed that duration of mucosa observation is strongly associated with adenoma detection in the proximal colon. The correlation between observation time and ADR sustained after controlling for common confounders such as age, gender and comorbidities. Moreover we showed that the impact of time on ADR is stronger in the proximal part compared to the distal part of the colon. Particularly in screening patients observation time was significantly associated with adenoma detection in the proximal colon. Thus data provided from our study suggest that the time factor plays a key role in the detection of proximal adenomas.

The use of a magnetic endoscope imaging device enabled us to control the actual scope position throughout the procedure. With the help of the MEI system polyps could be assigned to either the proximal or distal colon. MEl usage furthermore allowed us to record exact time spans for polyp detection in both the proximal and distal colon. We believe that the results derived from our study can be considered valid information in terms of the impact of the time effect on side-specific adenoma detection.

Some polyp features have been shown to cause problems in detecting proximal lesions during colonoscopy. One major issue in this context is polyp morphology. It has previously been shown that in particular small or flat proximal adenomas may be missed during withdrawal [18]. This finding is of crucial importance as small lesions in the proximal colon more frequently contain advanced neoplasia compared to the distal part [19]. In this study we found significant differences among polyp morphology in the distal and proximal colon. Even though the distribution of flat and diminutive lesions was equal between both sides, we found a significantly higher number of pedunculated polyps in the distal part. It is understandable that flat lesions are less easily detected than protruding or pedunculated polyps.

In our trial there was also a trend towards a higher share of serrated lesions in the proximal colon. These lesions also have been reported to be at special risk of being missed during

- Table 4 Impact of observation time on adenoma detection according to logistic regression analysis.

\begin{tabular}{|c|c|c|c|c|}
\hline & & OR & $95 \% \mathrm{Cl}$ & $P$ value \\
\hline \multirow[t]{2}{*}{ Proximal colon } & Non-adjusted & 3.643 & $2.370-5.599$ & $<0.001$ \\
\hline & Adjusted* & 3.610 & $2.283-5.709$ & $<0.001$ \\
\hline \multirow[t]{2}{*}{ Distal colon } & Non-adjusted & 1.818 & $1.145-2.888$ & 0.011 \\
\hline & Adjusted* & 2.341 & $1.395-3.929$ & 0.001 \\
\hline
\end{tabular}


screening [20]. We assume that a slower withdrawal enhances the chance of detecting polyps that are naturally difficult to find. The same reasoning could apply for the rather weak correlation between distal observation time and adenoma detection, which was documented in our trial. A surplus of time will not be per se necessary for the detection of lesions that can be identified easily.

It is also important to note, that polyps harvested in the proximal colon more often revealed adenomatous histology compared to distal polyps. In the proximal part $75 \%$ of all polyps were adenomas whereas only $45 \%$ showed adenomatous histology in the distal part. In terms of colorectal cancer prevention our findings emphasize the importance of polyp detection and resection particularly in the proximal colon.

It is well known that individual adenoma detection rates differ among colonoscopists [21-23]. We obtained a broad spectrum of personal ADRs that ranged between $24 \%$ and $50 \%$. It would be interesting to investigate which role the observation time plays in terms of personal detection rates. It would be conceivable that some investigators reach good ADRs in a shorter investigation period while others would not improve adenoma detection even while increasing the observation time. However in this trial the amount of colonoscopies contributed by each colonoscopist differed considerably. Due to low amounts of investigations contributed by each individual investigator we decided not to correlate personal detection rates with personal observations times. However, the problem should be addressed in future studies.

\section{The optimal investigation time in the proximal colon}

Barclay et al. first published their breaking results on the impact of withdrawal time on ADR in 2006 [24]. Since then a withdrawal time of at least 6 minutes has been implemented broadly as one out of several quality markers for colonoscopy. However some publications recently raised the hypothesis that a 6 minutes time span probably would be not long enough. In a recent study Jover showed that an overall withdrawal time of $>8$ minutes was associated with both proximal and distal adenoma detection. The effect of a longer withdrawal (> $8 \mathrm{~min}$ ) surpassed the one that could be demonstrated for the conventional 6 minutes time span [25]. Our data show that a sufficient time span is essential for proximal adenoma detection. In consequence it would be reasonable to demand for a specific period of time in the proximal colon, analogous to the 6 -minute overall benchmark. We found a minimum proximal observation time of at least 4 minutes to ensure sufficient adenoma detection in our trial. Thus, a 4-minute time span should be regarded as a lower limit which should not be fallen short of. However, data derived from this study showed that the probability of adenoma detection increases when a time span of more than 4 minutes is used. This fact raises the question of how long colonoscopists should spend for observation in the proximal colon. The latter question cannot be answered accurately on the basis of our data. However, the results should encourage endoscopists to use their time available bearing in mind that an increase in time enhances the chance of detecting more adenomas in the proximal colon.

Our study was not primarily designed to examine observation time in the distal or in the entire colon. However, a total time span of 6 minutes seems to be a short period of time considering that 4 minutes should be reserved for the proximal coIon. Future studies are needed in order to specifically investigate the impact of observation time on distal adenoma detection. It has to be mentioned that the named time span measured in our trial was a net duration of screening for polyps. We achieved the time values by subtracting time that was not technically spent for detection. This method has been used in studies on the same subject before [26]. However observation time in this case cannot be equated to withdrawal time. It could be argued that stopping the net time would not be a practicable during every-day practice. However we decided to use an approach that would give us the most valid information for the question of interest.

\section{Limitations}

Several limitations should be mentioned. First, this is a single center trial, and results may therefore not be generalizable. Second, we cannot definitively exclude bias that might derive from the detection of polyps itself. It is conceivable that the detection of the first polyp (regardless of polyp histology) would enforce the endoscopist to lower the withdrawal speed during the rest of the procedure. This in turn would enhance the chance to detect adenomas. In order to eliminate this uncertainty, it would be necessary to know the exact point in time of adenoma detection during withdrawal. Unfortunately, this information was not available in our trial. Thirdly it must be stated that colonoscopists were not blinded for the time measurement intervention. The awareness that observation time was measured using a stopwatch could have led to a slower withdrawal speed in this trial. Fourthly this was not a pure screening collective. The fact that patients in this trial underwent colonoscopy for a multitude of reasons may have distorted the results. In addition, results were obtained at a tertiary referral center. Investigations therefore may have been particularly demanding from a technical point of view. The latter fact may have resulted in relatively long cecal intubation time spans entailing the chance to detect adenomas also during insertion. However no significant impact of a prolonged CIT on ADR could be shown according to the regression analysis. Finally, it has to be mentioned that MEI devices are not routinely available in endoscopy units. Without the use of MEI is may be difficult to identify the exact border of the proximal colon and to adhere to a minimum time span in the respective segment.

\section{Conclusion}

In summary, results derived from our study show that time is a crucial factor for the detection of proximal adenomas during colonoscopy. The duration of observation has a stronger impact on adenoma detection in the proximal colon compared to the distal colon. A 4-minute time span was found to be the minimal requirement for the detection of adenomas in the 
proximal colon. We therefore propose to spend at least 4 minutes for screening in this colon segment.

\section{Acknowledgements}

The authors thank Olympus Germany for providing Evis Exera III CF-HQ 190 colonoscopes and processors for the duration of the study.

\section{Competing interests}

None

\section{References}

[1] Ryerson AB, Eheman CR, Altekruse SF et al. Annual Report to the Nation on the Status of Cancer, 1975-2012, featuring the increasing incidence of liver cancer. Cancer 2016; 122: 1312 -1337

[2] Marshall DC, Webb TE, Hall RA et al. Trends in UK regional cancer mortality 1991 - 2007. Br J Cancer 2016; 114: 340 - 347

[3] Robert Koch Institute and the Association of Population-based Cancer Registries in Germany (2014). In Internet: www.krebsdaten.de/Krebs/ EN/Content/Publications/Cancer_in_Germany/cancer_chapters_2009_2010/cancer_germany_2009_2010.pdf?__blob = publicationFile (Accessed 2016 August 18)

[4] Nakagawa $\mathrm{H}$, Ito $\mathrm{H}$, Hosono $\mathrm{S}$ et al. Changes in trends in colorectal cancer incidence rate by anatomic site between 1978 and 2004 in Japan. Eur J Cancer Prev 2017; 26: 269-276

[5] Samadder N], Curtin K, Pappas L et al. Risk of Incident Colorectal Cancer and Death After Colonoscopy: A Population-based Study in Utah. Clin Gastroenterol Hepatol 2016; 14: 279-86.e1-2

[6] Hewett DG, Rex DK. Miss rate of right-sided colon examination during colonoscopy defined by retroflexion: an observational study. Gastrointest Endosc 2011; 74: 246-252

[7] Chandran S, Parker F, Vaughan R et al. Right-sided adenoma detection with retroflexion versus forward-view colonoscopy. Gastrointest Endosc 2015; 81: 608-613

[8] Shah SG, Saunders BP, Brooker JC et al. Magnetic imaging of colonoscopy: an audit of looping, accuracy and ancillary maneuvers. Gastrointest Endosc 2000; 52: 1 -8

[9] Bladen JS, Anderson AP, Bell GD et al. Non-radiological technique for three-dimensional imaging of endoscopes. Lancet 1993; 341: 719 722

[10] German Cancer Society, German Cancer Aid, AWMF, Evidenced-based Guideline for Colorectal Cancer, version 1.1 (2014). In Internet: leitlinienprogramm-onkologie.de/uploads/tx_sbdownloader/LL_Colorectal_Cancer_1.1_english.pdf (Accessed 2016 August 17)
[11] Klare P, Ascher S, Hapfelmeier A et al. Patient age and duration of colonoscopy are predictors for adenoma detection in both proximal and distal colon. World J Gastroenterol 2015; 21: 525 - 532

[12] R Core Team, R: A language and environment for statistical computing. R Foundation for Statistical Computing (2014). In Internet: www. R-project.org/ (Assessed 2017 July 5)

[13] Rondonotti E, Andrealli A, Amato A et al. Technical interventions to increase adenoma detection rate in colonoscopy. Expert Rev Gastroenterol Hepatol 2016; 10: 1349-1358

[14] Rex DK, Schoenfeld PS, Cohen J et al. Quality indicators for colonoscopy. Gastrointest Endosc 2015; 81: 31-53

[15] Rembacken B, Hassan C, Riemann JF et al. Quality in screening colonoscopy: position statement of the European Society of Gastrointestinal Endoscopy (ESGE). Endoscopy 2012; 44: 957 - 968

[16] Cooper GS, Xu F, Barnholtz SloanjS et al. Prevalence and predictors of interval colorectal cancers in medicare beneficiaries. Cancer 2012; 118: $3044-3052$

[17] Gervaz P, Usel M, Rapiti E et al. Right colon cancer: Left behind. Eur ] Surg Oncol 2016; 42: 1343-1349

[18] Hazewinkel Y, Tytgat KM, van Leerdam ME et al. Narrow-band imaging for the detection of polyps in patients with serrated polyposis syndrome: a multicenter, randomized, back-to-back trial. Gastrointest Endosc 2015; 81: $531-538$

[19] Gupta N, Bansal A, Rao D et al. Prevalence of advanced histological features in diminutive and small colon polyps. Gastrointest Endosc 2012; 75: $1022-1030$

[20] IJspeert JE, van Doorn SC, van der Brug YM et al. The proximal serrated polyp detection rate is an easy-to-measure proxy for the detection rate of clinically relevant serrated polyps. Gastrointest Endosc 2015; 82: $870-877$

[21] Bretagne JF, Ponchon T. Do we need to embrace adenoma detection rate as the main quality control parameter during colonoscopy? Endoscopy 2008; 40: $523-528$

[22] Hetzel JT, Huang CS, Coukos JA et al. Variation in the detection of serrated polyps in an average risk colorectal cancer screening cohort. Am J Gastroenterol 2010; 105: 2656-2664

[23] Bretagne JF, Hamonic S, Piette $C$ et al. Variations between endoscopists in rates of detection of colorectal neoplasia and their impact on a regional screening program based on colonoscopy after fecal occult blood testing. Gastrointest Endosc 2010; 71: 335-341

[24] Barclay RL, Vicari J], Doughty AS et al. Colonoscopic withdrawal times and adenoma detection during screening colonoscopy. N Engl J Med 2006; 355: 2533 - 2541

[25] Jover R, Zapater P, Polanía E et al. Modifiable endoscopic factors that influence the adenoma detection rate in colorectal cancer screening colonoscopies. Gastrointest Endosc 2013; 77: 381-389.e1

[26] Lee RH, Tang RS, Muthusamy VR et al. Quality of colonoscopy withdrawal technique and variability in adenoma detection rates (with videos). Gastrointest Endosc. 2011; 74: 128 -134 
- Supplement 1 Demographic, Clinical, and Procedural Characteristics of patients excluded from the primary analysis due to incomplete colonoscopy procedures.

\begin{tabular}{|l|c|}
\hline Factor & \\
\hline Age (y), mean (SD) & $63.2(11.9)$ \\
\hline Male sex & $11(55.0 \%)$ \\
\hline Body mass index $\left(\mathrm{kg} / \mathrm{m}^{2}\right)$, mean (SD) & $25.9(5.0)$ \\
\hline Smoking (current and ex) & $3(15.0 \%)$ \\
\hline Alcohol abuse & $5(25.0 \%)$ \\
\hline Diabetes mellitus & $5(25.0 \%)$ \\
\hline ASA class & \\
\hline - 1 & $5(25.0 \%)$ \\
\hline - 2 & $9(45.0 \%)$ \\
\hline - 3 & $6(30.0 \%)$ \\
\hline Ambulatory patients & $11(55.0 \%)$ \\
\hline Indications & \\
\hline - Screening & $6(30.0 \%)$ \\
\hline - Grounds to suspect Tumor & $4(20.0 \%)$ \\
\hline - Gastrointestinal bleeding or Anemia & $4(20.0 \%)$ \\
\hline - Abdominal complaints & $4(20.0 \%)$ \\
\hline - History of colorectal polyps & $0(0 \%)$ \\
\hline - Surveillance after colorectal carcinoma & $2(10.0 \%)$ \\
\hline - Others & $0(0 \%)$ \\
\hline
\end{tabular}

Values are presented as (\%) unless otherwise noted.

ASA, American Society of Anesthesiologists; SD, standard deviation; $\mathrm{m}^{2}$, square meter; max, maximum; mg, milligram; min, minimum.
Supplement 2 Polyps detected in the proximal and distal colon.

\begin{tabular}{|c|c|c|}
\hline Factor & $\begin{array}{l}\text { Proximal } \\
\text { colon }\end{array}$ & $\begin{array}{l}\text { Distal } \\
\text { colon }\end{array}$ \\
\hline Number of polyps (total) & 288 & 250 \\
\hline - Adenomatous polyps & $217(75.3 \%)$ & $113(45.2 \%)$ \\
\hline - Non-adenomatous polyps & $71(24.7 \%)$ & $135(54.0 \%)$ \\
\hline - Serrated Adenomas & $19(6.6 \%)$ & $8(3.2 \%)$ \\
\hline - Carcinomatous polyps & 0 & 2 \\
\hline Large polyps ( $\geq 10 \mathrm{~mm}$ ) & $33(11.5 \%)$ & $35(14.0 \%)$ \\
\hline Small polyps (6-9mm) & $56(19.4 \%)$ & $37(14.8 \%)$ \\
\hline Diminutive polyps ( $\leq 5 \mathrm{~mm})$ & $199(69.1 \%)$ & $178(71.2 \%)$ \\
\hline Mean polyp size (mm), mean (SD) & $5.2(4.5)$ & $5.5(6.0)$ \\
\hline $\begin{array}{l}\text { Pedunculated polyps (Paris class } \\
0 \text {-Ip) }\end{array}$ & $5(1.7 \%)$ & $18(7.2 \%)$ \\
\hline Sessile polyps (Paris class 0-Is) & $168(58.3 \%)$ & $139(55.6 \%)$ \\
\hline $\begin{array}{l}\text { Flat elevated polyps (Paris class } \\
0 \text {-Ila) }\end{array}$ & $103(35.8 \%)$ & $84(33.6 \%)$ \\
\hline Flat polyps (Paris class 0 -IIb) & $11(3.8 \%)$ & $7(2.8 \%)$ \\
\hline $\begin{array}{l}\text { Cases with at least } 1 \text { polyp } \\
\text { (side-specific PDR) }\end{array}$ & $169(35.2 \%)$ & $160(33.8 \%)$ \\
\hline $\begin{array}{l}\text { Cases with at least } 1 \text { adenoma } \\
\text { (side-specific ADR) }\end{array}$ & $133(27.7 \%)$ & $88(18.3 \%)$ \\
\hline
\end{tabular}

